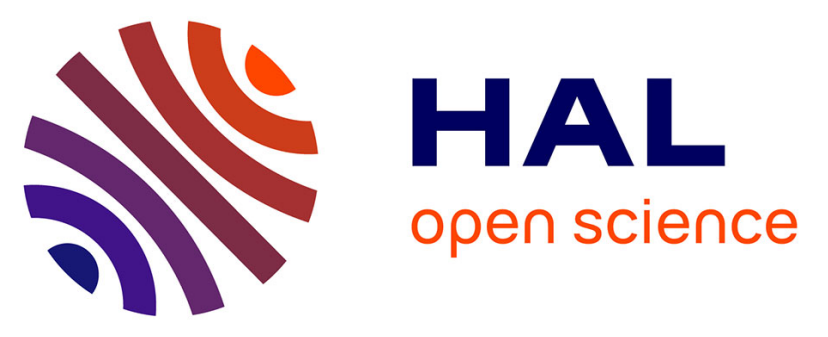

\title{
Chemical and Physicochemical Investigation of an Aminoalkylalkoxysilane As Strengthening Agent for Cellulosic Materials
}

\author{
Zied Souguir, Anne-Laurence Dupont, Jean-Baptiste D 'Espinose de \\ Lacaillerie, Bertrand Lavédrine, Hervé Cheradame
}

\section{To cite this version:}

Zied Souguir, Anne-Laurence Dupont, Jean-Baptiste D 'Espinose de Lacaillerie, Bertrand Lavédrine, Hervé Cheradame. Chemical and Physicochemical Investigation of an Aminoalkylalkoxysilane As Strengthening Agent for Cellulosic Materials. Biomacromolecules, 2011, 12 (6), pp.2082 - 2091. 10.1021/bm200371u . hal-01491189

\author{
HAL Id: hal-01491189 \\ https://hal.science/hal-01491189
}

Submitted on 22 Mar 2017

HAL is a multi-disciplinary open access archive for the deposit and dissemination of scientific research documents, whether they are published or not. The documents may come from teaching and research institutions in France or abroad, or from public or private research centers.
L'archive ouverte pluridisciplinaire HAL, est destinée au dépôt et à la diffusion de documents scientifiques de niveau recherche, publiés ou non, émanant des établissements d'enseignement et de recherche français ou étrangers, des laboratoires publics ou privés. 


\title{
Chemical and Physicochemical Investigation of an Aminoalkylalkoxysilane As Strengthening Agent for Cellulosic Materials
}

\author{
Zied Souguir, ${ }^{\dagger}$ Anne-Laurence Dupont, ${ }^{*,+}$ Jean-Baptiste d'Espinose de Lacaillerie, ${ }_{\varsigma}$ \\ Bertrand Lavédrine, ${ }^{\dagger}$ and Hervé Cheradame ${ }^{\S}$ \\ ${ }^{+}$Centre de Recherche sur la Conservation des Collections, Muséum National d'Histoire Naturelle, CNRS USR 3224, 36, \\ rue Geoffroy-Saint-Hilaire, F-75005, Paris \\ ${ }^{\ddagger}$ Laboratoire de Physico-chimie des Polymères et des Milieux Dispersés, Ecole Supérieure de Physique et de Chimie Industrielles, \\ CNRS UMR 7615, 10 Rue Vauquelin, F-75231 Paris cedex 05 \\ ${ }^{\S}$ Laboratoire Analyse et Modélisation pour la Biologie et l’Environnement, CNRS UMR 8587, Université d’Evry, \\ Boulevard François Mitterrand, F-91025 Evry cedex
}

Supporting Information

ABSTRACT: AMDES (aminopropylmethyldiethoxysilane) was used to investigate the physicochemical and chemical events related to the introduction of aminoalkylalkoxysilanes in cellulosic materials. Using ${ }^{29} \mathrm{Si} \mathrm{CP}-\mathrm{MAS}$ and ${ }^{1} \mathrm{H}$ NMR to study the reactivity and structural modification of AMDES in the paper it was shown that polymerization occurs in situ. The distribution of the active compound on the surface of the fibers and throughout the fibers' thickness was visualized by SEM-

EDS. A relation between moisture content, fiber swelling, and uptake of AMDES was found. To better represent old and brittle documents, the paper was predegraded by oxidation with sodium hypochlorite. XRD confirmed the advanced destruction of the amorphous areas of cellulose. Adding AMDES in the oxidized paper resulted in improved mechanical properties, a roughly unmodified degree of polymerization of cellulose, but a slight increase in the yellowing, probably due to several possible reaction products such as imines, amine, amides, and Maillard reactions products. The deacidification efficacy was established and the strengthening effect was shown to arise from the interaction of AMDES with the cellulose fibers.

\section{INTRODUCTION}

In libraries and archives some of the items that have acidified upon aging since their production are so brittle that they cannot be handled without risking loss of material. The main cause of decay of paper is its acid content in combination with time of exposure. Deacidification is the term used for a chemical treatment in paper conservation, which involves the neutralization of the acids present in the paper and the deposition of an alkaline compound such as calcium carbonate (often referred to as "alkaline reserve") to prevent, or at least delay, further acidification. Mass scale deacidification processes are commercially available for libraries and archives in several countries. ${ }^{1,2}$ However, institutions may be hesitant to resort to mass deacidification for the very acidic and brittle documents as these processes do not impart mechanical strengthening to the paper. A new chemical process that would simultaneously deacidify, introduce an alkaline reserve in the paper, and improve its mechanical properties would fulfill this purpose. To obtain the desired effects, we used aminoalkylalkoxysilanes. These molecules are used for the formation of hybrid materials, the immobilization of biomolecules or the modification of surface activity. ${ }^{3-6}$ Alkoxysilanes have also been proposed for the reinforcement of natural fibers. A review of the literature shows that most of these alkoxysilanes are tri- and tetra-functional and that they form interpenetrated networks. ${ }^{7-9}$ These networks of synthetic polymers with natural fibers can result in composite materials. The experimental conditions of solvent, and temperature used to produce these materials however are too drastic to be applied to historic documents.

In previous publications, a new solvent phase process based on aminoalkylalkoxysilanes (AAAS) was proposed for use in libraries and archives. ${ }^{10-13}$ Besides a clear improvement of the mechanical resistance of the paper, the treatment was shown to enhance stability toward aging processes and confer fungistatic properties to paper. ${ }^{11-13}$ Nevertheless, it was observed that for very oxidized and brittle papers the efficiency of the treatment was less satisfactory. ${ }^{13}$ In this work, a fundamental study was undertaken to better comprehend the reasons of this limitation. The understanding of the chemical and physicochemical events

\footnotetext{
Received: January 17, 2011

Revised: April 19, 2011

Published: April 20, 2011
} 


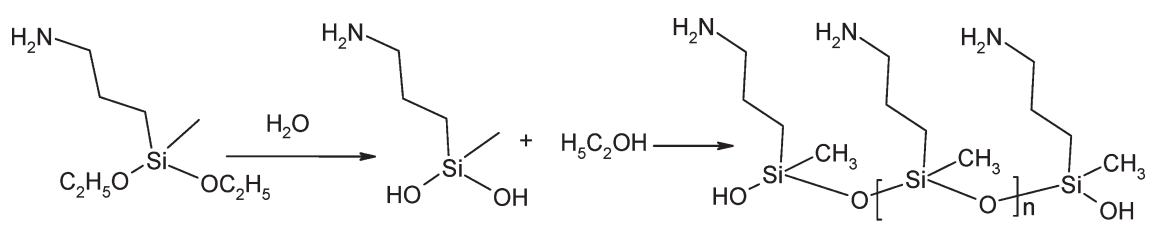

AMDES
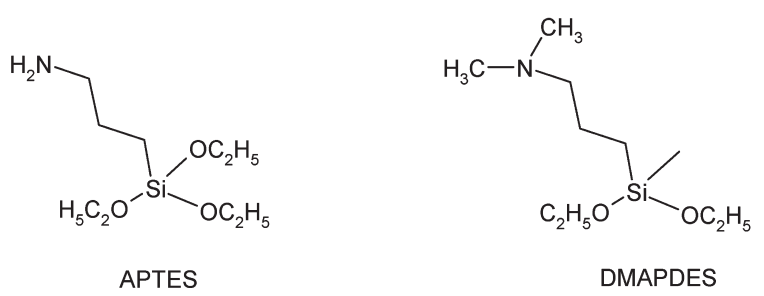

Figure 1. Top: 3-Aminopropylmethyldiethoxysilane (AMDES) and its reaction path (hydrolysis and condensation) to form poly-3-aminopropylmethylsilanediol (poly-AMSdiol). Bottom: Dimethylaminopropylmethyldiethoxysilane (DMAPDES) and 3-Aminopropyltriethoxysilane (APTES).

occurring between cellulose macromolecules and AAAS is essential in the interpretation of their effect as strengthening agents of cellulosic materials and to tailor treatment molecules specifically adapted to papers in different conservation conditions. Aminopropylmethyldiethoxysilane (AMDES), which has been studied in detail previously, ${ }^{14}$ was chosen due to its capability to polycondensate as a linear polymer upon hydrolysis.

Several physical parameters which are thought to influence the uptake of AMDES in the paper, and the reactivity of the AAAS toward cellulose, such as contact time, effect of residual humidity, and fiber swelling were investigated in a pure cellulose paper (P2). The polymerization of AMDES in situ in the paper was characterized using NMR and its degree of penetration in the fibers by SEM-EDS. The physicochemical and mechanical properties of P2 were then studied before and after its chemical oxidation to various degrees with sodium hypochlorite $(\mathrm{NaClO})$ at neutral $\mathrm{pH}$. This oxidation, which leads also to chain length reduction, was performed to achieve a degree of degradation that would be somewhat more representative of old and brittle papers.

\section{EXPERIMENTAL SECTION}

Materials. 3-Aminopropylmethyldiethoxysilane (AMDES) has one amine functionality and one silicon atom bearing two ethoxy groups. The polycondensation of the silanol functions resulting from hydrolysis can form linear (and cyclic) oligomers and polymers (Figure 1). Dimethylaminopropylmethyldiethoxysilane (DMAPDES) and 3-aminopropyltriethoxysilane (APTES) are also represented in Figure 1.

The "P2" paper used is made of cotton linters (>95\%) with traces of softwood pulp, and contains no fillers or sizing. Its basis weight is $76 \mathrm{~g}$ $\mathrm{m}^{-2}$, and it has a cold extract $\mathrm{pH}$ of 6.2. $\mathrm{P} 2$ was especially produced industrially in 1990 for the European project STEP.

The solvent used was hexamethyldisiloxane (HMDS). The concentration of the treatment solutions of AMDES in HMDS was 10\% (wt/ wt) unless otherwise stated. HMDS and all the AAAS were purchased from ABCR, Gelest.

Methods. The moisture content of the papers (MC; \% wt/wt) was determined according to TAPPI standard $\mathrm{T} 412 \mathrm{om}-02$ scaled down to a $50 \mathrm{mg}$ mass of paper. The AMDES uptake in the paper (\% wt/wt) was measured by weighing the samples preconditioned at $23{ }^{\circ} \mathrm{C}$ and $50 \%$ relative humidity $(\mathrm{rH})$ before and after treatment. The reported values are the average of the measurements on at least three samples. The alkaline reserve $(\mathrm{AR})\left(\mathrm{meq}\left(\mathrm{OH}^{-}\right) / 100 \mathrm{~g}\right)$ was determined by back-titration according to the standard method ASTM D4988-96R01. The cold extract $\mathrm{pH}$ of the papers was determined according to TAPPI T509 om-88, scaled down to a $50 \mathrm{mg}$ mass of paper.

The viscometric average degree of polymerization of cellulose $\left(\mathrm{DP}_{v}\right)$ was measured according to the standard method ISO 5351 with the solvent cupriethylenediamine (CED). To minimize alkaline degradation of oxidized papers in $\mathrm{CED},{ }^{15,16}$ samples underwent reduction in sodium borohydride, $0.01 \mathrm{M}$ in ethanol, during $17 \mathrm{~h}$ prior to the viscosity measurement. After reduction, the paper was thoroughly rinsed in water. Measurements were carried out in duplicate, and only the average values are reported.

The intrinsic viscosity of a dilute solution of a polymer is related to its molar mass. The relation is described by the empirical Mark-HouwinkSakurada equation

$$
[\eta]=K\left[\overline{M_{v}}\right]^{a}
$$

The relationship between the intrinsic viscosity $[\eta]$ and the degree of polymerization $\left(\mathrm{DP}_{v}\right)$ of cellulose in CED has been formulated as ${ }^{17}$

$$
[\eta]=0.91\left[\overline{\mathrm{DP}_{v}}\right]^{0.85}
$$

The value of $\mathrm{DP}_{v}$ is related to the number-average degree of polymerization $\mathrm{DP}_{n}$ by the expression

$$
\left[\overline{\mathrm{DP}_{v}}\right]=\left[\overline{\mathrm{DP}_{n}}\right] \times[(1+a) \Gamma(1+a)]^{1 / a}
$$

where $a$ is the Mark-Houwink coefficient for the cellulose/CED system, and $\Gamma(a+1)$ is the gamma function of $a$ evaluated by reference to tabulated values. ${ }^{18}$

Size-exclusion chromatography with multiangle light scattering and differential refractive index detection (SEC-MALS-DRI) was carried out for the determination of the average molar masses of cellulose, using $0.5 \%$ lithium chloride/dimethylacetamide ( $\mathrm{LiCl} / \mathrm{DMAc}$ ) as eluent, according to a method published previously where the paper is dissolved in $8 \% \mathrm{LiCl} / \mathrm{DMAc} .{ }^{19}$ Only the untreated papers were analyzed with SEC-MALS-DRI, as previous experience with AAAS led to column clogging. SEC-MALS-DRI was done to verify that the relation used to calculate $\mathrm{DP}_{n}$ was valid over the whole range of DP. Each sample solution was run three times nonconsecutively.

The copper number $\mathrm{N}(\mathrm{Cu})\left(\mathrm{g} \mathrm{Cu}_{2} \mathrm{O}\right)$, which represents an index for compounds in paper that possess reducing properties (such as carbonyl functions), ${ }^{20}$ was determined according to TAPPI standard T $430 \mathrm{~cm}-99$, with a reduced mass of paper of $300 \mathrm{mg}$. Three to nine repeat measurements were done for each sample type, and only the average value is given.

Tensile modulus at break ( $\mathrm{MPa}$ ) and elongation at break (\%) were measured with a Adamel Lhomargy instrument (DY-20B), according to 
the standard method NF: Q03-004 July 1986, where a coaxial stress is applied to the paper sample. Samples were tested at a speed of $10 \mathrm{~mm}$ $\min ^{-1}$, with the 100 decaNewton (DaN) load cell. The data were processed with TestWorks 4 (MTS Systems Corp.) software.

Zero-span tensile strength (zsTS) was measured with a Pulmac instrument (TS 100) according to TAPPI standard T231 cm-96. When measured on samples as conditioned, it is referred to as dry zsTS. The measurement carried out after saturating the paper sample with water for $1 \mathrm{~min}$ prior to mounting in the pulling jaws is referred to as wet zsTS. Wetting the paper allows to eliminate interfiber bonding, and intrinsic fiber resistance alone to be measured. The value obtained $(P)$ is transformed applying the formula zsTS $=\left(P-P_{0}\right) \times 0.372(\mathrm{~kg}-$ force $/ 15 \mathrm{~mm})$ modified to $\left(P-P_{0}\right) \times 5.64\left(\mathrm{daN} \mathrm{mm}^{-1}\right)$, where $P_{0}=2$ (constant of the instrument).

Folding endurance (FE; log of number of double folds) was determined with a Tinius Olsen double fold instrument, according to ISO 5626:1993. The applied force was $0.5 \mathrm{~kg}$.

These mechanical properties were measured in the machine direction of the paper, on 10 strips taken from the same sample conditioned at $23^{\circ} \mathrm{C}$ and $50 \% \mathrm{rH}$. Reported values are the average of the 10 measurements.

Color measurements were carried out with a hand-held spectrophotometer SP 64 (X-rite) equipped with an integrating sphere. The configuration adopted was in reflectance mode (spectral range $400-700 \mathrm{~nm}$ in $10 \mathrm{~nm}$ steps), with the specular component included, using the $5 \mathrm{~mm}$ diameter aperture. The colorimetric coordinates values $(\mathrm{L}, \mathrm{a}, \mathrm{b})^{*}$ were calculated in the CIE*Lab76 Color System, with the D65 Standard Illuminant and $10^{\circ}$ Standard Observer. ${ }^{21}$ Based on the $(\mathrm{L}, \mathrm{a}, \mathrm{b})^{*}$ values measured before and after a treatment, the total color change $\left(\Delta E^{*}=\left(\left(\Delta L^{*}\right)^{2}+\left(\Delta a^{*}\right)^{2}+\left(\Delta b^{*}\right)^{2}\right)^{1 / 2}\right)$ occurred on a given sample was calculated.

A variable pressure scanning electron microscope JEOL JSM-5410LV was used to examine the samples. The accelerating voltage was $20 \mathrm{kV}$ and the pressure was $0 \mathrm{~Pa}$. Samples were observed using backscattered electron imaging. The mapping of $\mathrm{Si}$ was done over the same area by energy dispersive X-ray spectrometry (EDS) by means of an Oxford Instruments Link PentaFET Si $(\mathrm{Li})$ detector and the software ISIS Link. The EDS operating conditions were as follows: accelerating voltage 20 $\mathrm{kV}$, ATM windows, take off angle $30^{\circ}$, working distance $20 \mathrm{~mm}$, count time $50 \mathrm{~s}$, approximate dead time $15 \%$. The samples were gold-coated before analysis.

${ }^{1} \mathrm{H}$ NMR was carried out on a Bruker Avance 300 instrument working at $300 \mathrm{MHz}$ with a frequency lock on deuterium. Liquid samples were introduced in $5 \mathrm{~mm}$ diameter tubes. Spectra were scanned at room temperature. $\left\{{ }^{1} \mathrm{H}-{ }^{29} \mathrm{Si}\right\}$ cross-polarization CP/MAS NMR was carried out on a Bruker ASX500 at 99.3 MHz (11.7 T). The ${ }^{1} \mathrm{H}$ pulse width $(\pi /$ 2) was $6 \mu \mathrm{s}$, the contact time $4 \mathrm{~ms}$, unless otherwise specified, and the recycle delay was $20 \mathrm{~s}$. The zirconia rotor of $4 \mathrm{~mm}$ diameter was spun at 5 $\mathrm{kHz}$. Chemical shifts were referred to an external reference, tetramethylsilane (TMS).

Thermogravimetric analysis in the temperature range $25-800^{\circ} \mathrm{C}$ was carried out using a TGA Q50 V6.7 Build 203 instrument.

The molecular organization of the cellulose component of the paper was characterized using X-ray diffraction. An XRD (Bruker D8 advance diffractometer, operated at $40 \mathrm{kV}, 40 \mathrm{~mA}$ ) was utilized to scan and record the data, using $\mathrm{Cu} \mathrm{K} \alpha$ radiation at $2 \theta=5-40^{\circ}$ with a step size of $0.04^{\circ}$. The crystalline index (CrI) of the cellulose samples was calculated using the following equation ${ }^{22}$

$$
\mathrm{CrI}=\frac{I_{002}-I_{\mathrm{am}}}{I_{002}} \times 100
$$

where $I_{002}$ is the peak intensity from the $(002)$ lattice plane $\left(2 \theta=22.6^{\circ}\right)$ and $I_{\mathrm{am}}$ the intensity of amorphous phases $\left(2 \theta=18.5^{\circ}\right)$.
Table 1. Uptake (\% wt/wt) of DMAPDES, AMDES, and APTES in P2 Papers

\begin{tabular}{ll} 
DMAPDES & $2 \pm 1$ \\
AMDES & $10 \pm 1$ \\
APTES & $23 \pm 1$ \\
\hline
\end{tabular}

Crystallite size $(\mathrm{L}(002))$ was estimated using Scherrer equation ${ }^{23}$

$$
L(002)=\frac{0.9 * \lambda}{\beta^{*} \cos \theta}
$$

where $\lambda$ is the wavelength of the incident $\mathrm{X}$-ray ( $\left.1.5418 \mathrm{~A}^{\circ}\right), \theta$ is the Bragg angle corresponding to the (002) plane, and $\beta$ is the half-height width of the peak angle of the (002) reflection.

AMDES Impregnation of the Papers. A simple system was designed to ensure reproducibility of the uptake from sample to sample, not only from one experiment to the next, but also within the same experiment. The impregnation was carried out by immersing four paper sheets (about $12 \mathrm{~g}$ ) separated with synthetic nonwoven support fabric and placed on a metallic grid in the treatment solution $(1 \mathrm{~L})$ containing $10 \%$ aminosilane in HMDS (wt/wt) at room temperature under magnetic stirring for the desired length of time. This procedure allowed reproducibly impregnating four sheets of paper. After treatment, the sheets were dried under vacuum for $1 \mathrm{~h}$ at room temperature. Control papers were not subjected to any treatment as it was shown that the immersion in HMDS did not modify the paper's mechanical properties.

\section{RESULTS AND DISCUSSION}

Chemical Structure of the Aminosilane versus Uptake. Three aminoalkylalkoxysilanes, namely, dimethylaminopropylmethyldiethoxysilane (DMAPDES), aminopropylmethyldiethoxysilane (AMDES), and aminopropyltriethoxysilane (APTES), were used to evaluate the relation between the chemical structure of the AAAS and the subsequent uptake in the paper. DMAPDES is a tertiary amine, whereas AMDES is a primary amine, and both are difunctional silanes. APTES is a primary amine but a trifunctional silane. Upon hydrolysis of their ethoxy functions, unlike the difunctional silanes, which polymerize as linear (or cyclic) molecules, trialkoxysilanes polymerize in three-dimensional structures and can build large silicon polymer networks. This explains the high uptake of 23\% APTES in the paper while AMDES provides a more reasonable uptake of $10 \%$ (Table 1). Conversely, with DMAPDES, the uptake is negligible. This was attributed to the nature of the amine functionality. As opposed to primary amines, tertiary amines are likely less adsorbed by hydrophilic surfaces like cellulose and cannot form hydrogen bonds with cellulose. The results support the interpretation that the interactions between cellulose and aminoalkyldialkoxysilanes are favored by hydrogen bonds as proposed in previous work. $^{13}$

The SEM micrographs and EDS X-ray mappings of the cross sections of P2 treated with AMDES, APTES and DMAPDES show that in the three cases, the silicon atoms are localized across the thickness of the paper sheet, with heavier deposits on the fibers' surface but also clearly, throughout the interior of the fiber (Figure 2). This is particularly visible for AMDES and APTES. This result shows that aminoalkylalkoxysilanes tend to spread over the fibers but they can also penetrate inside through the cotton fiber wall. Previous research on aminosilanes reports the ability of these compounds for surface coating. ${ }^{7-9}$ In the present work, they appear as penetrants as well. The phenomenon of 

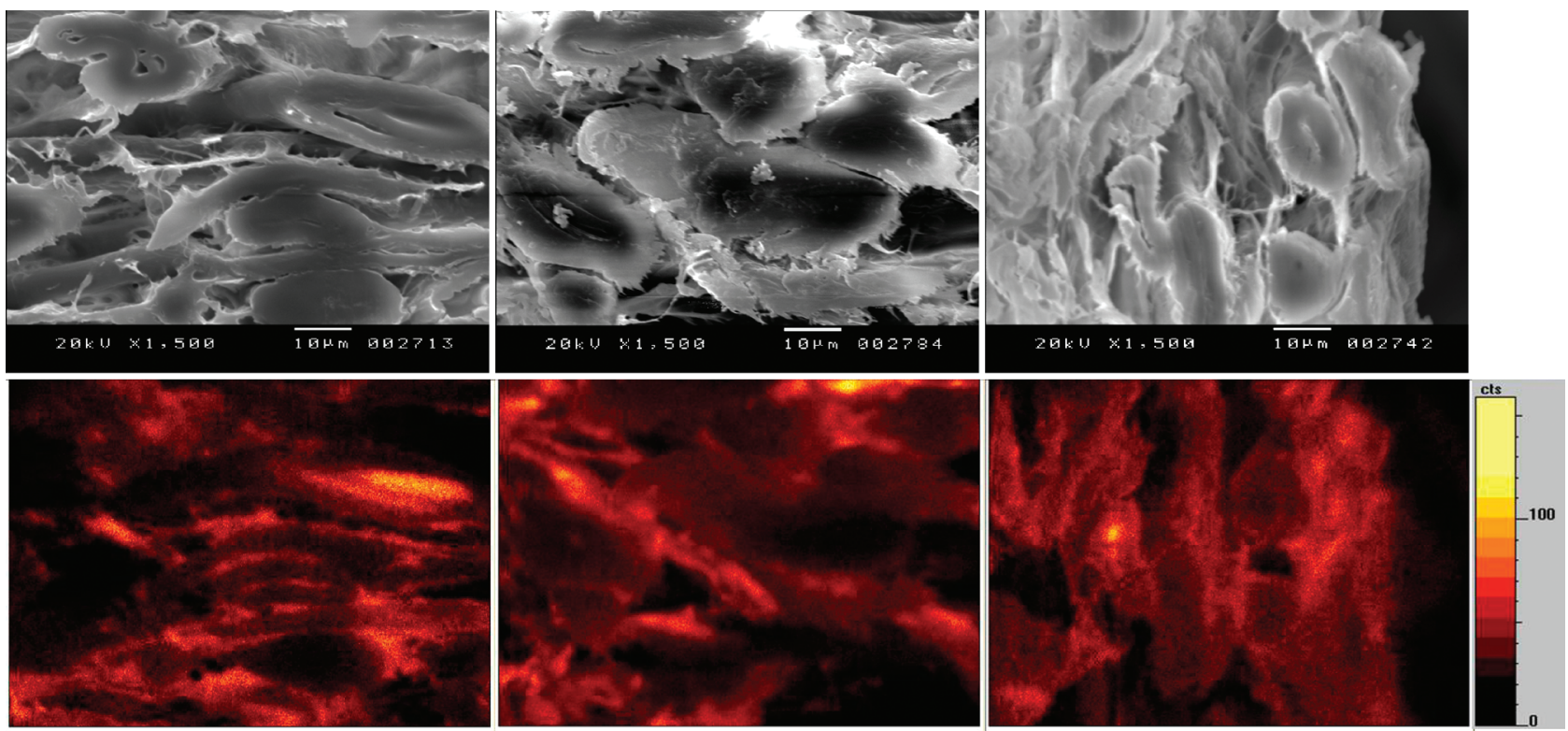

Figure 2. Top: SEM micrographs of cross sections of P2 treated with (A) AMDES, (B) APTES, and (C) DMAPDES. Bottom: EDS atomic mappings of $\mathrm{Si}$ (red) in corresponding areas.

penetration is most likely to be attributed to the impregnation method, which uses a complete immersion of the papers in the AAAS monomer/solvent solutions.

Figure 2 also shows that APTES aggregates are formed on the fibers' surface. In a previous study it was observed that treatment with APTES increased paper rigidity. ${ }^{11}$ Because DMAPDES does not provide sufficient uptake to ensure alkaline reserve, AMDES was chosen for the purpose of this study.

The SEM micrograph of a cross section of the reference paper P2 Ctrl together with those of P2 treated with the various AAAS indicate that the latter do not appear to modify the fiber morphology (Figure 2B in the Supporting Information).

Influence of Physical Parameters on the Uptake of AMDES. Effect of Contact Time. The determination of a suitable treatment time for a uniform impregnation was a preliminary requirement. Four sheets were placed in the treating solution for 30 and $60 \mathrm{~min}$. After drying, the uptakes were all in the range of $10 \pm$ $1 \%$. The duration of $30 \mathrm{~min}$ thus seemed sufficient for the solution to penetrate evenly inside the paper fibers' web. The same solution was used a second time for the impregnation of four new sheets, also for 30 and $60 \mathrm{~min}$. Again, the uptakes were all of $10 \pm 1 \%$. It was shown that the same solution could be used up to four times without a noticeable change in the uptake of AMDES in the papers.

The alkaline reserve measured on two sheets of $\mathrm{P} 2$ immersed in AMDES during 30 and $60 \mathrm{~min}$ was $44 \pm 4 \mathrm{meq}\left[\mathrm{OH}^{-}\right] / 100 \mathrm{~g}$ for both, which confirmed the result obtained from uptake measurements. It can be calculated that 44 meq $\left(\mathrm{OH}^{-}\right) / 100 \mathrm{~g}$ corresponds to an AMDES uptake of $8.4 \%$. This was confirmed by a thermogravimetric measurement where for P2 untreated, the final weight residue was negligible, whereas for P2 treated with AMDES, the final weight residue was of $3.6 \%$, which corresponds to the silica powder $\left(\mathrm{SiO}_{2}\right){ }^{24}$ The back-calculation of the initial uptake of AMDES in paper yields a value of $9.5 \%$, which is consistent with the value reported previously. The calculations were made under the assumption that ethoxy functions of AMDES are totally hydrolyzed, which will be demonstrated onward with ${ }^{1} \mathrm{H}$ NMR analysis.

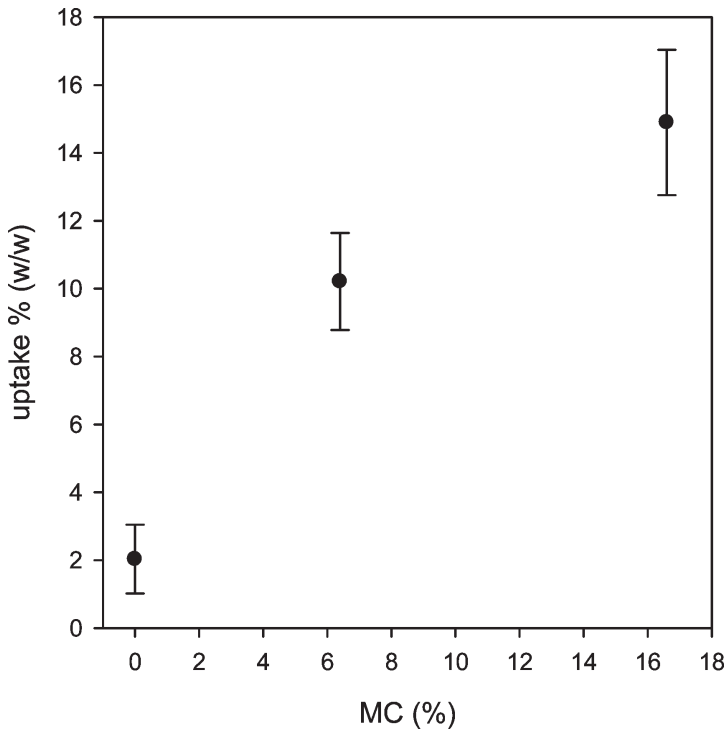

Figure 3. AMDES uptake variation as a function of $\mathrm{P} 2$ moisture content (MC).

Effect of Moisture Content and Fiber Swelling. A higher swelling of cellulose fibers eases the approach of inclusion compounds to the more accessible regions. ${ }^{25}$ The role of residual moisture content and additional water and the subsequent fiber swelling state during the impregnation with AMDES on the resulting properties of the paper was examined.

The AMDES uptake was measured in various conditions of moisture in the paper and dryness of the treatment solution. To dry the paper completely without promoting its degradation, sheets were placed in a vacuum oven for $24 \mathrm{~h}$ at $80^{\circ} \mathrm{C}$. HMDS previously dried on molecular sieves was mixed with AMDES in a glovebox under an argon atmosphere, and the paper was immediately immersed in the solution. 


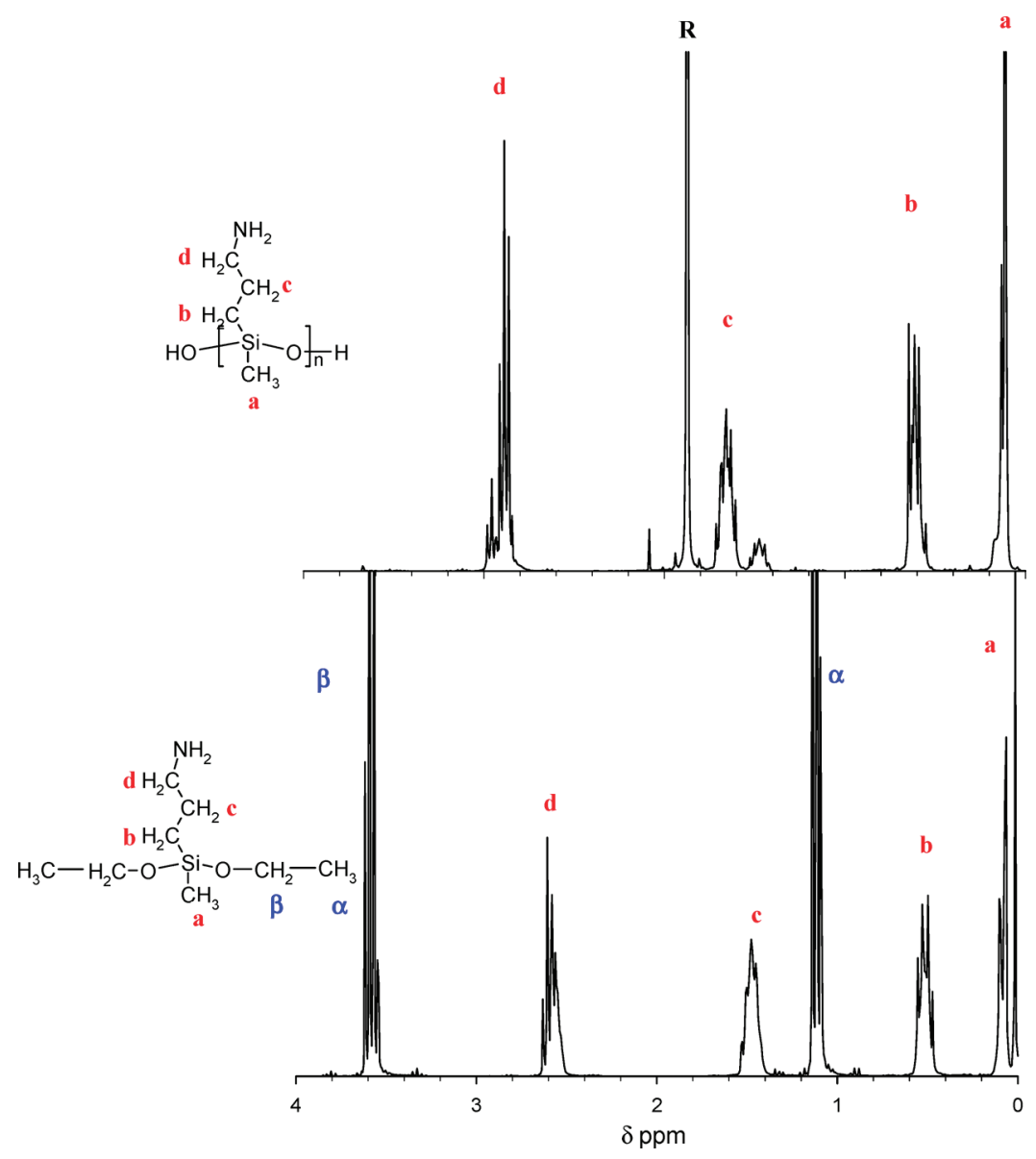

Figure 4. Top: ${ }^{1} \mathrm{H}$ NMR spectrum of a $\mathrm{D}_{2} \mathrm{O}$ extract of $\mathrm{P} 2$ treated with AMDES in presence of sodium acetate (R) as an internal reference. Bottom: ${ }^{1} \mathrm{H}$ NMR spectrum of AMDES dissolved in $\mathrm{D}_{2} \mathrm{O}$.

Water vapor saturation conditions were obtained by placing the paper in a hermetically closed vessel in the presence of water for $72 \mathrm{~h}$ at room temperature, thus creating conditions of $100 \%$ $\mathrm{rH}$. The sheets were then immediately treated with AMDES.

Solvent saturation conditions were obtained by immersing paper sheets in HMDS previously dried on molecular sieve, followed by their drying under vacuum for $1 \mathrm{~h}$ and immediate treatment with AMDES.

Figure 3 shows the variation of the AMDES uptake as a function of the paper moisture content at $23{ }^{\circ} \mathrm{C}$ for the dehydrated papers (MC 0\%), the papers conditioned at $50 \%$ $\mathrm{rH}(\mathrm{MC} 6.4 \%)$ and the papers conditioned at $100 \% \mathrm{rH}(\mathrm{MC}$ $16.6 \%)$. The water content seems to play an important role in the AMDES uptake. The latter varied from $2 \pm 1 \%$ for the dehydrated paper to $14.9 \pm 2.1 \%$ for the paper saturated with water vapor. The small uptake observed in the case of the dry paper could be due to the adventitious presence of residual moisture during the experiment or to simple adsorption of AMDES on the fibers surface.

In this system, the underlying mechanism of the effect of water in paper is thus physical, water plays a physical role as a plasticizer by swelling the cellulose fibers. However, water can also react chemically leading to the hydrolysis of the ethoxy functions of AMDES, and their replacement by silanol functions, eventually followed by condensation reactions. This point is studied in the following paragraph.
Study of the Reactivity of AMDES toward Cellulose Using ${ }^{7} \mathrm{H}$ and ${ }^{29} \mathrm{Si}$ CP MAS NMR Spectroscopy. $\mathrm{A} \mathrm{D}_{2} \mathrm{O}$ extract of AMDEStreated paper was prepared with $0.9732 \mathrm{~g}$ paper in $13.228 \mathrm{~g} \mathrm{D}_{2} \mathrm{O}$. For a quantitative determination of the portion of AMDES extracted from the paper, a known quantity $(0.0245 \mathrm{~g})$ of sodium acetate trihydrate was added to a known quantity $(1.057 \mathrm{~g})$ of the sample solution. Sodium acetate was used as an internal standard to compare the peak areas (R) $1.87 \mathrm{ppm}\left(\mathrm{CH}_{3}-\mathrm{CO}_{2}\right)$ and (a) 0.1 ppm $\left(\mathrm{CH}_{3}-\mathrm{Si}\right)$. The peaks integration showed that about $80 \%$ of AMDES was extracted from the sample. This shows that the AMDES is easily removed from the paper by immersion in water. This was confirmed by SEM-EDS. The simultaneous Si mapping of two samples treated with AMDES, one of which was subsequently washed in water evidenced a very small amount of $\mathrm{Si}$ in the latter (data not shown).

The ${ }^{1} \mathrm{H}$ NMR spectra of this sample and that of AMDES in $\mathrm{D}_{2} \mathrm{O}$ are shown in Figure 4. It can be observed that the characteristic peaks of ethoxy groups, which are visible on the ${ }^{1} \mathrm{H}$ NMR spectrum of the solution of AMDES in $\mathrm{D}_{2} \mathrm{O}\left((\alpha) \mathrm{CH}_{3}\right.$ : $\left.1.22 \mathrm{ppm},(\beta) \mathrm{CH}_{2}: 3.6 \mathrm{ppm}\right)$, are totally absent on the spectrum of the $\mathrm{D}_{2} \mathrm{O}$ extract of the paper treated with AMDES. However, on the latter spectrum, the $\mathrm{CH}_{2}$ multiplets of the propyl moiety are clearly visible at (b) 0.6 , (c) 1.6 , and (d) $2.9 \mathrm{ppm}$. This result shows that AMDES in the paper is completely hydrolyzed, the ethanol produced by this reaction being lost during the drying under vacuum. It has been shown in a previous publication that 

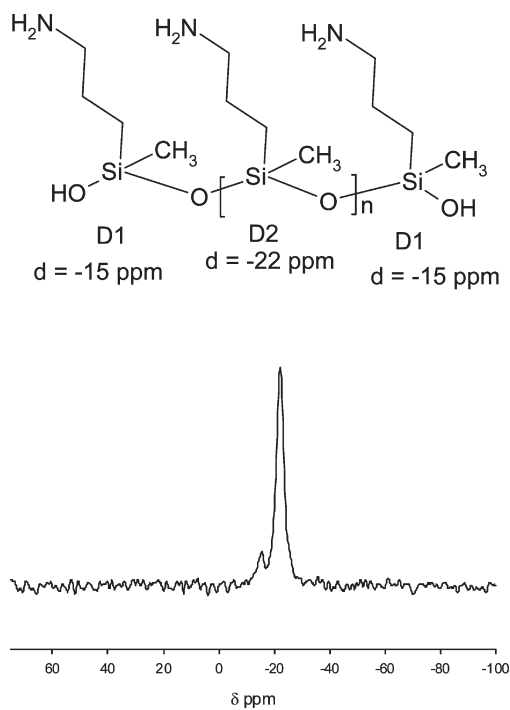

Figure 5. ${ }^{29} \mathrm{Si}$ CP MAS NMR spectrum at room temperature of P2 treated with AMDES.

AMDES can react directly with ethanol or methanol by an exchange reaction, even in anhydrous conditions. ${ }^{14}$ The same publication also showed that the reaction rate increased in the presence of water.

To determine whether the hydrolyzed molecules of AMDES can condensate in situ in the paper, solid state ${ }^{29} \mathrm{Si} \mathrm{NMR}$ spectroscopy with cross-polarization was used on a sample of P2 paper treated with AMDES and defibrillated in a two-blade blender. The ${ }^{29} \mathrm{Si}$ CP MAS NMR spectrum is shown on Figure 5. Two peaks are clearly visible around -15 and $-22 \mathrm{ppm}$. According to the literature, the peak at $-22 \mathrm{ppm}$ corresponds to a $\mathrm{D}_{2} \mathrm{Si}$, that is, a silicon atom linked to two $-\mathrm{O}-\mathrm{Si}$ substituents, while the peak at $-15 \mathrm{ppm}$ corresponds to a $\mathrm{D}_{1} \mathrm{Si}$, linked to only one $\mathrm{O}-\mathrm{Si}$ group, that is, a terminal silicon atom. ${ }^{26,27}$

In a recent study, hydrolysis and condensation of AMDES in methanol and ethanol were investigated using high resolution ${ }^{29} \mathrm{Si}$ NMR in liquid phase. The alcohol medium provides functional environment conditions similar to cellulose, which bears several $-\mathrm{OH}$ groups. It was shown that the silicon resonance of poly(3-aminopropylmethylsiloxane) appeared at $-22.05 \mathrm{ppm}$, that of the cyclic tetramer $\mathrm{D}_{4}$ appeared at $-19.5 \mathrm{ppm}$, and that of the cyclic trimer $\mathrm{D}_{3}$ at $-11.3 \mathrm{ppm} .{ }^{28,29}$ These chemical shifts are similar to those observed in the case of dimethyldiethoxysilane upon hydrolysis and condensation, for which silicon resonance of the cyclic $\mathrm{D}_{4}$ and of the cyclic $\mathrm{D}_{3}$ appeared at -19.1 and -8.7 ppm, respectively. ${ }^{29}$ Unfortunately, the resolution of the peak on the ${ }^{29} \mathrm{Si}$ CP MAS spectrum does not allow concluding that the cyclic tetramer was missing $(-19.5 \mathrm{ppm})$. However, despite significant noise, the cyclic trimer at $-8.7 \mathrm{ppm}$ is obviously absent from the spectrum. It is known that during the polymerization of dialkoxysilanes by polycondensation, linear and cyclic compounds among which are $\mathrm{D}_{4}$ and $\mathrm{D}_{3}$, are formed. In this case, the absence of $\mathrm{D}_{3}$ is an indication for the absence of $\mathrm{D}_{4}$. This observation supports the fact that the cyclization reaction between two terminal silanol groups in the polycondensation process of AMDES does not occur in the paper. This is most probably due to insufficient mobility of the two terminal groups. This has already been observed when the reaction is confined in a twodimensional space, that is, on a surface. Conversely, linear

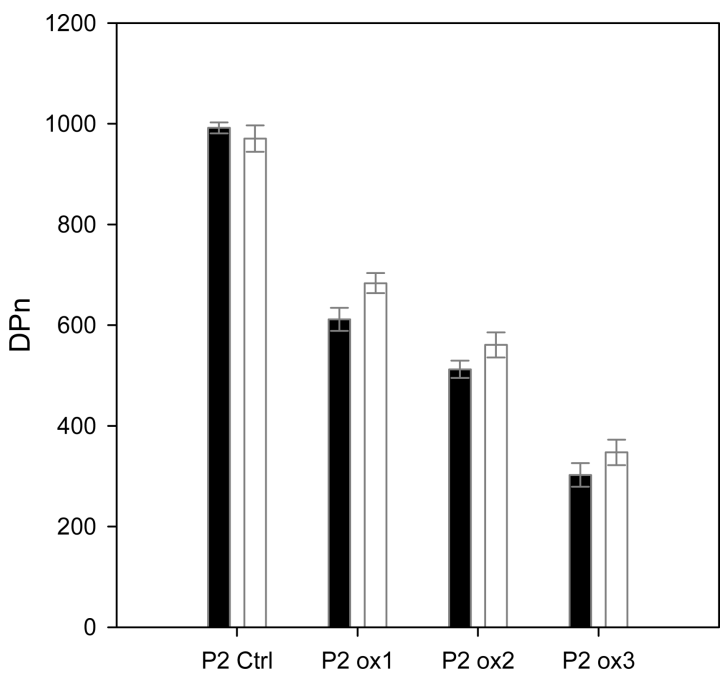

Figure 6. DP ${ }_{n}$ of P2 Ctrl and P2 oxidized ( $\square$ ) before treatment and ( $\square$ ) after treatment with AMDES.

oligomers can form in situ with a higher probability, by the reaction of terminal silanol functions of monomers in the paper with monomer molecules still in solution (Figure 1).

Although CP MAS NMR is a semiquantitative technique, the average AMDES polymers formed were composed of approximately 10 monomers, as calculated from the relative intensity of the two peaks on Figure 5. This relatively large DP is in agreement with the assumption of a polymerization mechanism involving freely moving monomer molecules able to react with terminal silanol groups on the fibers surface. Furthermore, the absence of a peak at $-4 \mathrm{ppm}$ corresponding to the $\mathrm{Si}$ of free AMDES monomers ${ }^{14}$ confirms the total polymerization.

Physicochemical and Mechanical Properties of Oxidized Paper Treated with AMDES. Effect of Oxidation at the Morphological and Macromolecular Level of Cellulose. Paper degradation occurs largely through acid hydrolysis and oxidation. Attack of cellulose with sodium hypochlorite $(\mathrm{NaClO})$ has been characterized to occur randomly in the accessible areas of the fibers and lead to considerable chain scission, as well as to the formation of carbonyl groups (aldehyde, ketone, and carboxyl groups) on C2, C3, and C6, and short chain organic acids. ${ }^{30,31}$ The nature and relative amount of the carbonyl functions created depend largely on the $\mathrm{pH}$ at which the oxidation is carried out. At neutral $\mathrm{pH}$, aldehyde and ketone functions are predominant, while in alkaline medium, carboxylic functions are primarily created on the cellulose macromolecules.

The oxidation was carried out by immersing P2 paper for 30 min in aqueous solutions of $\mathrm{NaClO}$ at $\mathrm{pH} 7$ (adjusted with $\mathrm{HCl} 6$ $\mathrm{N})$ at $0.26,0.39$, and $0.52 \%$ active chlorine. Four sheets were used in each case. After the oxidation, the sheets were thoroughly rinsed with deionized water to eliminate all residual chlorine, and dried in a ventilated hood at room temperature. The results are shown in Table 2 in the Supporting Information.

The values obtained for the copper number confirmed that the oxidation at $\mathrm{pH} 7$ created carbonyl functions on the cellulose, the quantity of which depended on the concentration of $\mathrm{NaClO} . \mathrm{DP}_{n}$ values obtained by viscometry and SEC-MALS-DRI for all the untreated samples analyzed were found rather similar, showing the validity of the relationship $\left([\eta]=0.91\left[\overline{\mathrm{DP}_{v}}\right]^{0.85}\right)$ and the relationship between $\mathrm{DP}_{v}$ and $\mathrm{DP}_{n}$ for highly degraded cellulose. 

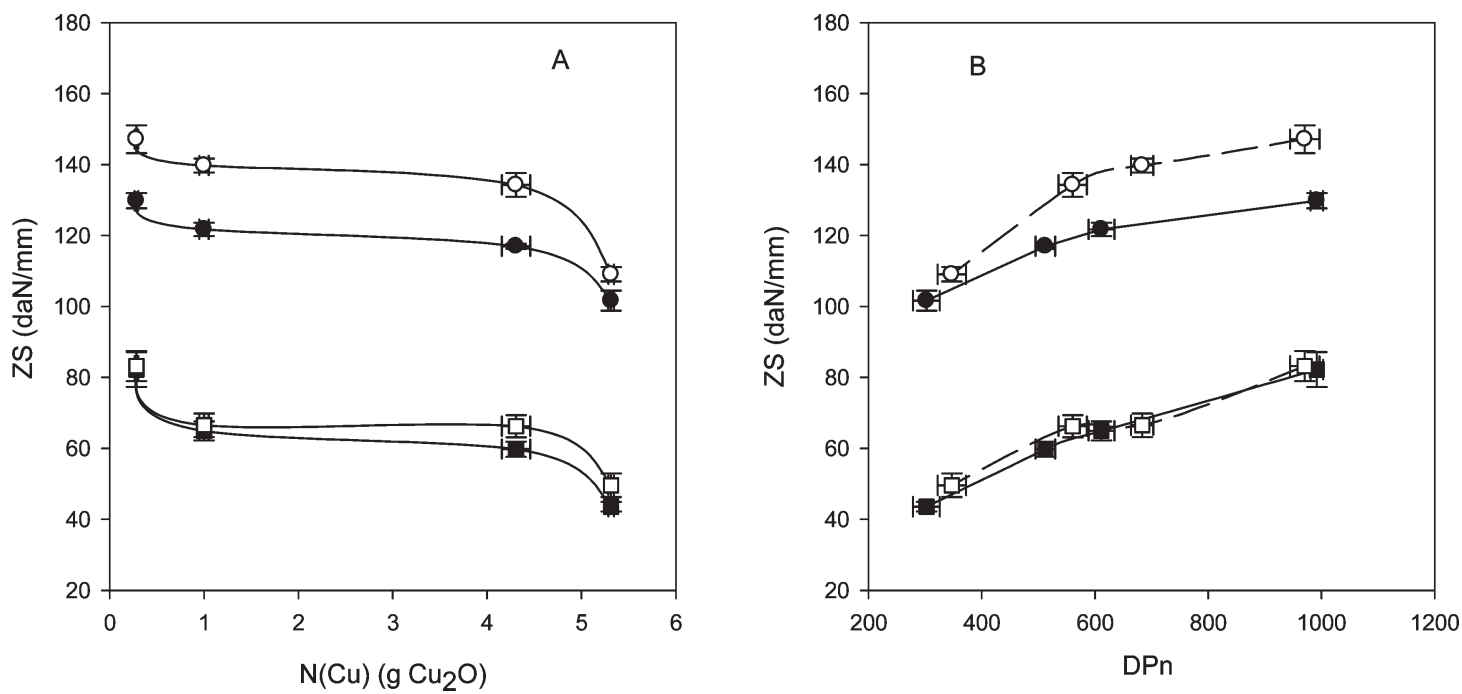

Figure 7. (A) Variation as a function of copper number $\mathrm{N}(\mathrm{Cu})$ of P2 Ctrl and P2 oxidized of dry zero span tensile strength (dry zsTS) ( $)$ before treatment and $(O)$ after treatment with AMDES, and wet zero span tensile strength (wet zsTS; $\mathbf{\square})$ before treatment and $(\square)$ after treatment with AMDES. (B) Variation as a function of $\mathrm{DP}_{n}$ of P2 Ctrl and P2 oxidized of dry zsTS $(-)$ before treatment and $(\mathrm{O})$ after treatment with AMDES, and wet zsTS $(\square)$ before treatment and $(\square)$ after treatment with AMDES.

The steep decrease in $\mathrm{DP}_{n}$ (also illustrated in Figure 6) indicates that the oxidation reaction at $\mathrm{pH} 7$ induced extensive cleavage of the $\beta(1-4)$ glycosidic bond of cellulose, which is consistent with published data. ${ }^{32}$

The degree of crystallinity of the cellulose measured using XRD for the sample with a copper number of 5.31 (P2 ox3) showed a slight increase compared to P2 Ctrl. The oxidation induced a preferential removal of the amorphous areas of the cellulose. This was confirmed by the unchanged size of the crystallites after the oxidation reaction. Also, as expected, oxidized paper showed a lower moisture content than nonoxidized paper. This decrease is due to the degradation of the amorphous areas in the cellulose fibers which, unlike the crystalline areas, can accommodate water molecules.

Effect of AMDES Treatment on the Physicochemical Properties of Oxidized Paper. The same treatment procedure as for nonoxidized papers was used for the oxidized papers. The data in Table 3 in the Supporting Information show that oxidized papers have a smaller AMDES uptake than the control paper. This could result from the smaller moisture content in the paper (Table 2), as it has been shown above that moisture content does play a role in the AMDES uptake (Figure 3). A high proportion of carbonyl groups on cellulose would also hinder hydrogen bonding with AMDES.

Upon oxidation the cold extract $\mathrm{pH}$ of the papers remained quite similar, the low molar mass water-soluble acids produced during the oxidation being washed away by the water rinsing. As expected, the $\mathrm{pH}$ of the aqueous extract of the papers treated with AMDES increased considerably and to the same alkaline value regardless of the uptake, due to the amine functionality, implying a deacidification of the paper and the deposition of an alkaline reserve.

One drawback observed was that the treatment brought some discoloration to the paper ( $b^{*}$ aft values). Although the total color change was not perceptible in the case of P2 $\operatorname{Ctrl}\left(\Delta E^{*}<1\right),{ }^{21}$ it was slightly noticeable $\left(1<\Delta E^{*}<3\right)$ for all the oxidized papers. This might be attributable to the reaction of the amine groups of AMDES with the carbonyl groups on cellulose, forming imine, amine, or amide functions as in Maillard reactions. ${ }^{33-35}$
Figure 6 shows $\mathrm{DP}_{n}$ of $\mathrm{P} 2$ as a function of oxidation before and after treatment with AMDES. Although not to be considered statistically significant, a small increase of $\mathrm{DP}_{n}$ for the treated papers compared with untreated papers was observed for all the samples. This could possibly be attributed to the chemical reactions between oxidized cellulose and AMDES as explained above. This hypothesis is consistent with the decrease in copper number and the increase in yellowing observed after the treatment of the paper (Table 3 ). It could also merely be an apparent increase in $\mathrm{DP}_{n}$ as considering that such reactions take place, the calculation of the $\mathrm{DP}_{v}$ using the Mark-Houwink coefficients for pure cellulose may not be applicable. At any rate the important conclusion from this experiment is that the treatment with AMDES did not cause macromolecular degradation to cellulose.

Effect of AMDES Treatment on the Mechanical Properties of Oxidized Paper. The different mechanical properties (zsTS, modulus at break, elongation at break, and folding endurance) of treated and untreated papers were measured. The relation between the changes in mechanical properties and the physicochemical characteristics of cellulose ( $\mathrm{DP}_{n}$ and copper number) were then studied.

Figure 7A shows the evolution of dry and wet zero span tensile strength versus copper number for P2 Ctrl and P2 oxidized, before and after treatment with AMDES. As expected, the dry zero-span tensile strength of the oxidized paper decreased, which is due to the degradation of the cellulose chains. For the treated samples, the same variation, that is, a decrease of zero-span tensile strength with increasing $\mathrm{N}(\mathrm{Cu})$, was observed. However, for the oxidized samples, the nominal values of dry zsTS of these treated samples were all higher than the values for their untreated counterpart. It can be assumed that the interfiber and intrafiber bonding of cellulose fibers was improved by the presence of the AMDES oligomers. Conversely, the difference between the wet zero-span tensile strength of the untreated and the treated samples at equal oxidation stage was much smaller, with these values being all lower than the dry zsTS values. As upon the wetting necessary to perform the measurement in the wet state, most of the AMDES is solubilized in the water, this shows that 

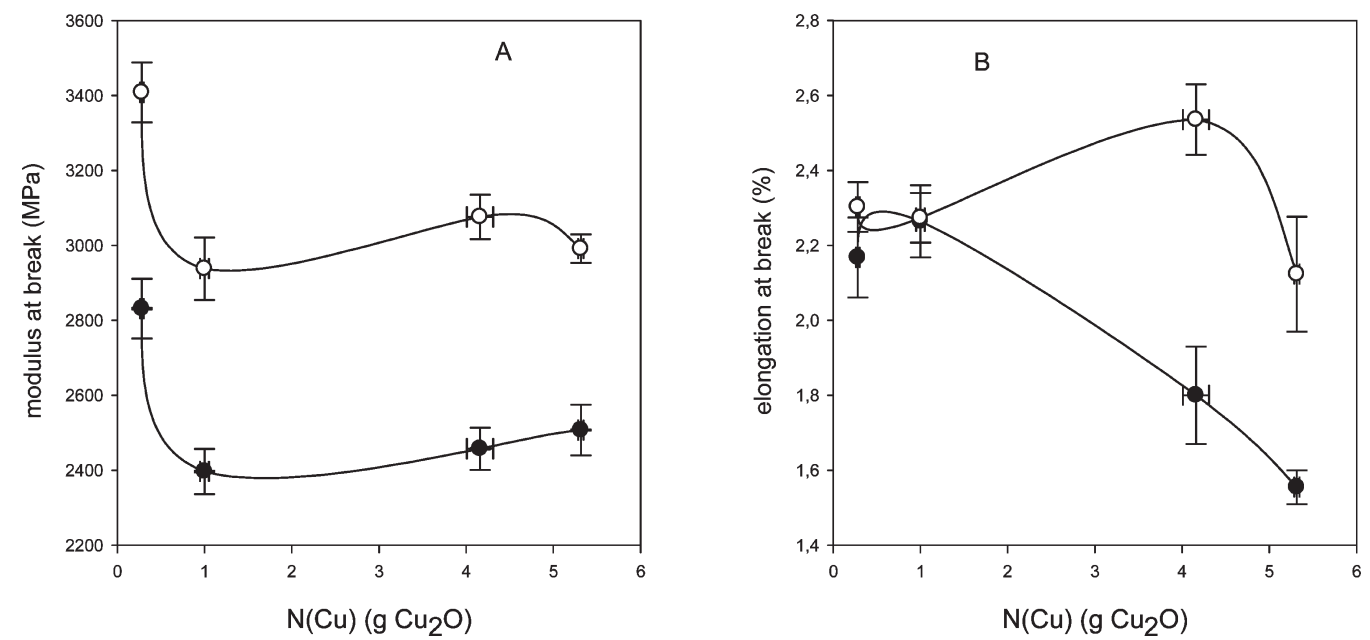

Figure 8. (A) Variation of the modulus at break as a function of the copper number $\mathrm{N}(\mathrm{Cu})$ for P2 $\mathrm{Ctrl}$ and $\mathrm{P} 2$ oxidized $(\bullet)$ before and $(\mathrm{O})$ after AMDES treatment. (B) Variation of the elongation at break as a function of $\mathrm{N}(\mathrm{Cu})$ of P2 Ctrl and P2 oxidized $(\mathbf{O})$ before and $(\mathrm{O})$ after AMDES treatment.
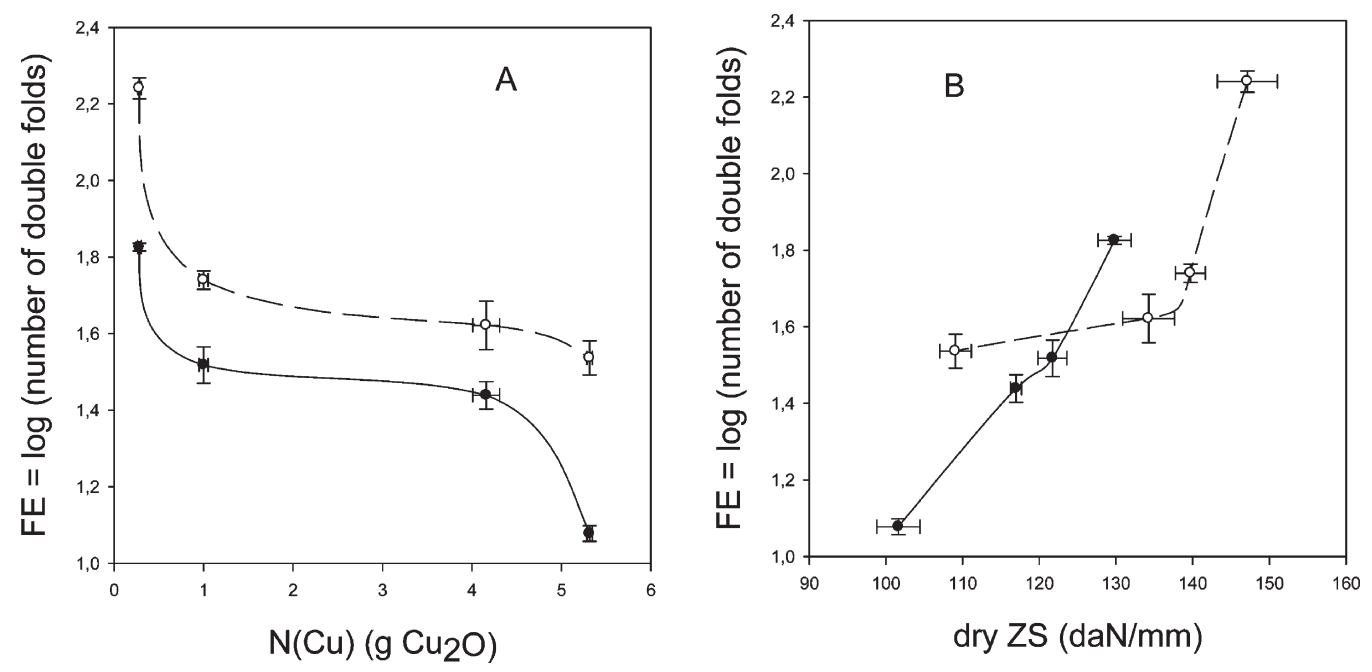

Figure 9. (A) Folding endurance as a function of the copper number $\mathrm{N}(\mathrm{Cu})$ of $\mathrm{P} 2 \mathrm{Ctrl}$ and $\mathrm{P} 2$ oxidized $(\bullet)$ before and $(\mathrm{O})$ after AMDES treatment. (B) Folding endurance as a function of dry zero-span tensile strength (dry zsTS) of P2 and P2 oxidized (O) before and (O) after AMDES treatment.

the intrinsic resistance of the fibers was not modified by the presence of the residual AMDES oligomers. Indeed, it was verified that zsTS measurements for the AMDES treated paper subsequently washed in water and dried yield the same value as for P2Ctrl in the dry state (in the order of $128 \pm 8 \mathrm{daN}$ $\mathrm{mm}^{-1}$ ). These results tend to indicate that the strengthening effected by AMDES is probably due to hydrogen bonding with cellulose fiber. This is consistent with the result shown earlier that only AAAS that are able to form hydrogen bonding have the capacity to be incorporated in the fibers in significant amounts.

The curves of the dry zsTS as a function of $\mathrm{DP}_{n}$ for both treated and untreated papers show the typical profile, where zsTS is largely unaffected by the polymer chain length reduction on a large range of DP (at the higher DP end) and falls more rapidly below a certain DP value, which has been reported as "critical DP”36,37 (Figure 7B). Conversely, a quasi linear relationship between wet zsTS and $\mathrm{DP}_{n}$ was observed for both the treated and the untreated papers. Such a linear relationship had been observed before by Zou. ${ }^{38}$

Figure 8A shows the tensile modulus as a function of the copper number for the untreated and the AMDES treated papers. In both cases, a decrease was observed between P2 Ctrl and P2 oxidized. Then the modulus remained nearly constant upon increasing $\mathrm{N}(\mathrm{Cu})$ for both the treated and the untreated samples. However, it can be seen that the incorporation of AMDES in the paper increased the absolute values of the modulus, thus, the paper stiffness. This increase is consistent with the tensile strength improvement measured with zsTS. Accordingly, the elongation at break decreased dramatically for the oxidized papers (Figure $8 \mathrm{~B}$ ). The oxidation of the cellulosic material and the degradation of the amorphous areas led to increased rigidity. After treatment with AMDES, the elongation at break was roughly maintained for the moderately oxidized papers 
showing a better deformability capacity but decreased for highly degraded paper (Figure 8B).

The decrease of the folding endurance with increasing $\mathrm{N}(\mathrm{Cu})$ (Figure 9A) confirmed that oxidation greatly affected the paper strength while AMDES did impart somewhat better mechanical properties in terms of paper deformability.

In Figure 9B, the relation between the folding endurance and the dry zero-span tensile strength for papers untreated and treated with AMDES is presented. FE approximately followed the values of dry zsTS in a linear fashion for the untreated papers, showing that the folding endurance was proportional to the intrinsic strength of the fiber. For the treated papers, the curve followed a double regime. The nonoxidized and moderately oxidized samples showed a linear variation, and the slope of the curve was comparable to that of the untreated papers. In contrast, for the strongly oxidized papers, the folding endurance was roughly independent of the intrinsic strength of the fiber. This result shows that the folding endurance improvement differs depending on the original strength of the untreated fiber.

\section{CONCLUSION}

The analyses of treated paper carried out using ${ }^{1} \mathrm{H}$ NMR showed that a total hydrolysis of the ethoxy groups of the AMDES molecules occurs in the paper. This hydrolysis reaction is followed by a condensation reaction of the AMDES to yield linear oligomers exclusively, the cyclization process being hindered as shown by ${ }^{29} \mathrm{Si}$ CP-MAS NMR. The AMDES uptake was shown to be related with the moisture content in the paper and the fiber swelling state, with higher moisture leading to higher uptake. This is one advantage of the proposed treatment as current mass deacidification processes used in commercial scale often involve an initial drying step. ${ }^{1}$ From a point of view of cultural heritage preservation, it is worth limiting the number of pretreatment steps for old documents. Moreover, a process that would not require predrying would be more cost-efficient.

Using SEM-EDS on cross sections of papers, it was possible to visualize that AMDES was present on the fibers surface and throughout the fibers' interior. The penetration of the active compound through the fiber walls is a key parameter for the efficiency of the deacidification and strengthening effect and is an element of novelty compared to currently available protocols where the alkaline reserve deposits only on the fibers surface.

The oxidation of the paper with sodium hypochlorite was accompanied by the formation of carbonyl functions and by a large random cleavage of the cellulose chains. This degradation affected all accessible areas of the fibers, mainly the amorphous areas of the cellulose, and was accompanied by a loss of the mechanical properties of the paper. Upon incorporation of AMDES in oxidized papers the mechanical properties, especially in the dry state, were improved, with an increase in the zero-span tensile strength. The results in the wet state show that the partial removal of AMDES (inherent to the measurement) allows the paper to recover its initial zero-span tensile strength. The possible elimination of the AAAS from the paper, which was demonstrated with ${ }^{1} \mathrm{H}$ NMR and SEM-EDS, complies with one important requirement of conservation treatments of cultural heritage, which is their removability. An improvement of the elongation at break was shown, which was confirmed by the improved folding endurance. This can be attributed to the presence of the AMDES oligomers, which confer plasticity to the system.
One drawback that needs further investigation is the slight yellowing observed for the treated oxidized papers. A reaction between the amine of AMDES and the carbonyls on the cellulose possibly occurs, with formation of reaction products such as imines, amine, amides, and Maillard reactions products. This yellowing is quite limited and should not preclude the use of the new multipurpose mass treatment proposed for old and brittle paper documents in libraries and archives. However, only shortterm changes have been measured, and as yellowing can increase with paper aging, more tests need to be performed.

\section{ASSOCIATED CONTENT}

S Supporting Information. Additional data is presented in Tables 2 and 3 and Figure 2B. This material is available free of charge via the Internet at http://pubs.acs.org.

\section{AUTHOR INFORMATION}

\section{Corresponding Author}

*Tel.: +33 140795307. Fax: +33 140795312.E-mail: aldupont@ mnhn.fr.

\section{ACKNOWLEDGMENT}

A research grant from the French Ministry of Culture is gratefully acknowledged. Sabrina Paris, Eleonora Pellizzi, and Oulfa Belhadj from CRCC and Sid Labdi and Gilles Renou from $\mathrm{LAMBE}$ are warmly thanked for technical assistance.

\section{REFERENCES}

(1) Carter, H. A. J. Chem. Educ. 1996, 73, 417-420.

(2) Turko, K. Association of Research Libraries: Washington, D.C., 1990; p 33.

(3) Jacob, M.; Varughese, K. T.; Thomas, S. Biomacromolecules 2005, 6, 2969-2979.

(4) Pasqui, D.; Atrei, A.; Barbucci, R. Biomacromolecules 2007, $8,3531-3539$.

(5) North, S. H.; Lock, E. H.; Cooper, C. J.; Franek, J. B.; Taitt, C. R.; Walton, S. G. ACS Appl. Mater. Interfaces 2010, 2, 2884-2891.

(6) Moon, J. H.; Shin, J. W.; Kim, S. Y.; Park, J. W. Langmuir 1996, $12,4621-4624$.

(7) Bledzki, A. K.; Gassan, J. Prog. Polym. Sci. 1999, 24, 221-274.

(8) Belgacem, M. N.; Gandini, A. Compos. Interfaces 2005, 12, 41-75.

(9) Abdelmouleh, M.; Boufi, S.; Belgacem, M. N.; Duarte, A. P.; Salah, A. B.; Gandini, A. Int. J. Adhes. Adhes. 2004, 24, 43-54.

(10) Ipert, S.; Rousset, E.; Cheradame, H. Restaurator 2005, 26, $250-264$.

(11) Ipert, S.; Dupont, A. L.; Lavédrine, B.; Bégin, P.; Rousset, E.; Cheradame, H. Polym. Degrad. Stab. 2006, 91, 3448-3455.

(12) Rakotonirainy, M. S.; Dupont, A.-L.; Lavédrine, B.; Ipert, S.; Cheradame, H. J. Cult. Herit. 2008, 9, 54-59.

(13) Dupont, A.-L.; Lavédrine, B.; Cheradame, H. Polym. Degrad. Stab. 2010, 95, 2300-2308.

(14) Bennevault-Celton, V.; Maciejak, O.; Desmazières, B.; Cheradame, H. Polym. Int. 2010, 59, 1273-1281.

(15) Lee, S. B.; Feller, R. L.; Bogaard, J. J. Imaging Sci. 1985, 29, $61-64$.

(16) Strlič, M.; Kolar, J.; Žigon, M.; Pihlar, B. J. Chromatogr., A 1998, 805, 93-99.

(17) Evans, R.; Wallis, A. F. A. J. Appl. Polym. Sci. 1989, 37, 2331-2340.

(18) Davis, P. J. Gamma Function and Related Function. In Handbook of Mathematical Functions; Abramowitz, M, Stegun, I. A., Eds.; Washington, DC: National Bureau of Standards, 1972; pp 253-294. 
(19) Dupont, A.-L. Polymer 2003, 44, 4117-4126.

(20) Röhrling, J.; Potthast, A; Rosenau, T.; Lange, T.; Ebner, G.; Sixta, H.; Kosma, P. Biomacromolecules 2002, 3, 969-975.

(21) Marcus, R. T. The Measurement of Color. Color for Science, Art and Technology; Nassau, Ed.; Elsevier: Amsterdam, 1998; pp 31-96.

(22) De Souza, I. J.; Bouchard, J.; Methot, M.; Berry, R.; Argyropoulos, D. S. J. Pulp Paper Sci. 2002, 28, 167-170.

(23) Hindeleh, A. M.; Johnson, D. J. Polymer 1972, 13, 1327-1332.

(24) Wang, S.; Ahmad, Z.; Mark, J. E. Chem. Mater. 1994, 6, 943-946.

(25) Krässig, H. A. Cellulose: Structure, Accessibility and Reactivity, Gordon and Breach Science Publishers: New York, 1993.

(26) El Kortobi, Y.; d'Espinose de la Caillerie, J.-B.; Legrand, A.-P.; Armand, X.; Herlin, N.; Cauchetier, M. Chem. Mater. 1997, 9, 632-639.

(27) Lee, T.-M.; M. Ma, C.-C.; Hsu, C.-W.; Wu, H.-L. Polymer 2005, 46, 8286-8296.

(28) Brus, J.; Dybal, J. Polymer 2000, 41, 5269-5282.

(29) Zoldesi, C. I.; Van Walree, C. A.; Imhof, A. Langmuir 2006, 22, 4343-4352.

(30) Lewin, M.; Epstein, J. A. J. Polym. Sci. 1962, 58, 1023-1037.

(31) Potthast, A.; Rosenau, T.; Kosma, P. In Polysaccharides II. In Adv. Polym. Sci. 205; Klemm, D., Ed.; Springer-Verlag: Berlin, Heidelberg, 2006; pp 1-48.

(32) Whitmore, P. M.; Bogaard, J. Restaurator 1995, 16, 10-30.

(33) De la Orden, M. U.; Martínez Urreaga, J. Polym. Degrad. Stab. 2006, 91, 886-893.

(34) Martínez Urreaga, J.; De la Orden, M. U. Eur. Polym. J. 2006, 42, 2606-2616.

(35) Hodge, J. E. J. Agric. Food Chem. 1953, 1, 928-43.

(36) Zou, X.; Gurnagul, N.; Uesaka, T.; Bouchard, J. Polym. Degrad. Stab. 1994, 43, 393-402.

(37) Jerosch, H.; Lavédrine, B.; Cherton, J.-C. Restaurator 2002, 23, $222-239$.

(38) Zou, H. Effect of Kraft Pulping on Oxygen Delignification. Ph. D. dissertation, University of Maine, Orono, ME, 2002. 